\title{
Lumen
}

Selected Proceedings from the Canadian Society for Eighteenth-Century Studies

\section{Ma conversion, ou la puissance satirique du grotesque}

\section{Valérie Van Crugten-André}

Volume 15, 1996

URI : https://id.erudit.org/iderudit/1012483ar

DOI : https://doi.org/10.7202/1012483ar

Aller au sommaire du numéro

Éditeur(s)

Canadian Society for Eighteenth-Century Studies / Société canadienne d'étude du dix-huitième siècle

ISSN

1209-3696 (imprimé)

1927-8284 (numérique)

Découvrir la revue

Citer cet article

Van Crugten-André, V. (1996). Ma conversion, ou la puissance satirique du grotesque. Lumen, 15, 215-228. https://doi.org/10.7202/1012483ar d'utilisation que vous pouvez consulter en ligne.

https://apropos.erudit.org/fr/usagers/politique-dutilisation/ 


\section{Ma conversion, ou la puissance satirique du grotesque}

La littérature de l'époque (pré-)révolutionnaire et impériale a longtemps souffert du puissant discrédit qu'ont fait peser sur elle les historiens de la littérature. Négligeant de l'étudier en profondeur, ces derniers ont choisi de passer sous silence une période capitale de l'Histoire qui leur paraissait stérile et peuplée d'épigones. L'absence de chefs-d'oeuvre, les préjugés inspirés par le climat fin de siècle et la rupture révolutionnaire ont certes contribué à occulter l'existence d'une production dont le volume impressionne. Mais, Béatrice Didier l'a bien expliqué, c'est, à l'origine, une raison politique qui a motivé le désintérêt, voire le mépris, de la critique: 'L'histoire littéraire s'est constituée à partir de la Restauration, c'est-à-dire à un moment où l'on essaie d'oublier la Révolution, de fermer la parenthèse, de reprendre le fil de l'Ancien Régime. Et cette littérature, si elle révulse l'idéologie dominante, ne satisfait pas non plus les artistes: dans aucun des deux camps qui s'opposent alors - classique et romantique - elle ne peut susciter d'enthousiasme. ${ }^{1}$

Fort heureusement, on assiste depuis plusieurs années à une réhabilitation de la littérature de la fin des Lumières, à laquelle quelques spécialistes ont consacré de brillantes études. ${ }^{2}$ Ils ont mis en évidence la richesse d'une production abondante et diversifiée, permettant au chercheur de mieux cerner l'évolution des goûts esthétiques et des mentalités à l'aube de l'Epoque contemporaine.

Contrairement à ce qu'on pourrait croire, le genre romanesque jouit, dans les dernières années de l'Ancien Régime ainsi que sous la Révolution et $l^{\prime}$ Empire, d'une fortune considérable. Dans la Bibliographie $d u$ genre romanesque français, A. Martin, R. Frautschi et V.G. Mylne en fournissent la preuve irréfutable. On dénombre ainsi, entre 1780 et 1800, neuf cent soixante-et-un romans français nouveaux.

Les romans dits libertins, par exemple, dont on publie l'avis de décès après la parution des Liaisons dangereuses de Laclos en $1782,{ }^{3}$ se révèlent à l'examen un mort bien vivant! Bien sûr, ils ne sont plus tout à fait les mêmes. Certains ont mal vieilli, mauvais remakes des romans de Crébillon fils ou de Duclos, d'autres se sont transformés avec la société qui les 
a vus naître. Parmi ces derniers, les romans de Gabriel Honoré Riqueti, comte de Mirabeau, font figure d'exemple.

Mirabeau le tribun, dont l'éloquence, la laideur et les succès féminins sont devenus légendaires, le prisonnier de Vincennes et l'amant de Sophie, l'homme des célèbres discours ${ }^{4}$ est aussi l'auteur méconnu de textes libertins, remarquables par leur qualité et leur diversité. ${ }^{5}$ Nous avons choisi de nous arrêter ici sur l'un d'entre eux: Ma conversion, ou le libertin de qualité, publié pour la première fois en 1783.

Il ne s'agit pas là d'une décision arbitraire: pour la première fois dans le roman français (du moins le semble-t-il), ${ }^{6}$ le héros qui se raconte fait métier de se prostituer. En outre, Ma conversion permet de mettre en évidence la fascinante ambiguité de Mirabeau. Aristocrate et révolutionnaire, monarchiste et progressiste, il se situe à la charnière des Lumières et de l'avènement de la bourgeoisie.

Avec Ma conversion, il porte un regard lucide sur son époque, mêlant humour et dérision, cynisme et parodie; il ne veut pas changer la société (comme ce sera le cas dans ses écrits politiques), il la tourne en ridicule, pour mieux la dévoiler et en dénoncer les vices.

Mirabeau est en prison quand il compose Ma conversion en 1780. Il est enfermé au château de Vincennes, sur l'ordre de son père 7 qui éprouvera toute sa vie pour ce fils singulièrement laid et tellement différent une insurmontable aversion. Cette fois, il lui reproche sa dissipation, ses dettes et l'enlèvement de Sophie de Monnier dont Gabriel était l'amant. ${ }^{8}$

Ces circonstances pénibles ont souvent porté les critiques à considérer l'oeuvre érotique de Mirabeau comme un simple délassement de prisonnier, une catharsis où s'expriment les fantasmes d'un homme dont on connaît le tempérament et l'appétit sexuel.

S'il est vrai que l'auteur lui-même considérait Ma conversion comme un passe-temps agréable dans cet univers carcéral, il n'en affirme pas moins le caractère novateur ('[...]c'est une folie singulièrement neuve, ${ }^{\prime 9}$ 'ce roman, qui est absolument neuf ${ }^{10}$ ) et la portée satirique de son roman ('c'est une bonne charge et un vrai livre de MORALE,'11 'des peintures très-libres (sic), mais très-vraies (sic), de nos moeurs, de notre corruption, de notre libertinage'). ${ }^{12}$

Malgré les apparences, la portée du texte dépasse le grotesque et la farce: 'ce roman $[. .$.$] est moins frivole que l'on ne croirait au premier$ coup d'oeil,' déclarera-t-il lui-même dans une lettre à Sophie de Monnier. ${ }^{13}$

En quelques mots, donnons le ton du roman. Le narrateur, jeune libertin de 24 ans, décide de renoncer au plaisir gratuit de la conquête et de se faire gigolo des femmes sur le retour. Véritable prostitué, il passe en revue ses clientes et donne une description circonstanciée de ses durs travaux, qu'il interrompt çà et là de dialogues fictifs avec son interlo- 
cuteur (Satan), et de réflexions personnelles. Il décide de se marier, pour faire une fin et... alimenter ses rentes. Trompé sur la marchandise - la jeune épouse ne l'a pas attendu pour goûter les plaisirs de la chair et porte en elle le fruit de sa débauche... - il quitte le pays et son cocuage et décide de 'foutre la nature entière[...] jusqu'à ce que rendant dans les bras paternels de M. Satan [son] âme célibataire, [il] aille foutre chez les morts. ${ }^{14}$

Une première lecture suffit à mettre en évidence la satire sociale qui se cache derrière la bouffonnerie: Ma conversion est avant tout un roman du dévoilement, une peinture de moeurs émaillée d'ironie, $d$ 'humour et de cynisme.

Nous nous attacherons tout particulièrement à cet aspect de l'oeuvre, nous efforçant de mettre en évidence les différents motifs qui l'articulent, les procédés littéraires et linguistiques qui le renforcent.

La clef de voûte de Ma conversion est sans aucun doute le motif de l'argent. Présent de l'incipit à la fin du roman, il double la perspective satirique. En effet, nous sommes en présence de la parodie d'un genre littéraire (le roman libertin Ancien Régime) qui débouche sur la satire sociale proprement dite.

Le titre du roman (Ma conversion, ou le libertin de qualité), considéré dans ses rapports avec le texte entier, est particulièrement révélateur. Construit sur le modèle traditionnel des titres des romans de l'époque (un titre suivi d'un sous-titre qui l'explicite), il renferme déjà toute l'ironie qui scandera le texte.

Le mot conversion désigne le passage, l'adaptation d'un état à un autre, d'un système de valeurs à un autre. Mis en équation avec libertin de qualité, il est compris comme une transformation positive, une amélioration du protagoniste. Il nous semble erroné d'y voir, comme certain critique, ${ }^{15}$ une allusion au mariage final du héros, symbole d'un retour à la norme sociale et morale. Les circonstances de ce mariage et, surtout, les premières phrases du roman récusent cette interprétation.

L'incipit est retardé par l'épître dédicatoire à Satan, destinataire du récit. Cette introduction permet au lecteur de se faire une idée du ton du roman et contient déjà en germe la satire sociale qui suivra:

Mais, Monsieur Satan, tout est bien changé dans ce pays, vous devenez vieux; vous restez chez vous; les moines même ne peuvent vous en arracher. Vos diablereaux, pauvres hères! n'en savent pas autant que nos apprentis maquereaux; ils ne vous rapportent que des récits infidèles, parce que nos femmes les attrapent et les bernent.[...]; je vous offre mon livre. Vous y lirez la Gazette de la cour, les nouvelles à la main des filles, les financiers et les dévotes. Vous serez instruit de quelques tours de bissac où, tout fin diable que vous êtes, vous auriez eu un pied de nez. Mais que votre chaste épouse n'y fourre pas le 
sien, car aussitôt cornes de licornes s'appliqueraient sur votre front séraphique. [...] Puissent les tableaux que j'ai l'honneur de mettre sous vos yeux ranimer un peu votre antique paillardise! Puisse cette lecture faire branler tout l'univers! (37-38)

La société a tellement changé que le Mal lui-même est dépassé: l'élève a surpassé le maître ('Vous avez instruit mon adolescence,' (37)).

Après cette exhortation à la paillardise universelle (on a déjà ici un exemple de la démesure baroque dont nous aurons l'occasion de reparler plus avant), le texte s'ouvre sur cette profession de foi:

Jusqu'ici, mon ami, j'ai été un vaurien; j'ai couru les beautés; j'ai fait le difficile. A présent la vertu rentre dans mon coeur; je ne veux plus foutre que pour de l'argent; je vais m'afficher étalon juré des femmes sur le retour, et je leur apprendrai à jouer du cul à tant par mois (39).

La conversion est pour le moins saisissante. L'argent y règne en maître. Nous sommes en pleine parodie; la première phrase nous plonge dans la thématique traditionnelle du roman libertin: un débauché se repent et revient à la vertu. Or, pour le libertin de qualité, la vertu, c'est l'argent qui devient une valeur morale à part entière!

Tout a changé: le libertin Ancien Régime se vouait au Plaisir, le libertin de qualité voue un culte à Plutus et se prostitue. L'amour n'est plus une fin en soi mais un moyen; l'attrait de l'or lui fait surmonter son dégoût et le fait bander, le donjuanisme devient une industrie du plaisir vendu: ${ }^{16}$ ' [...] je pleure: l'or paraît... L'or! Sacredieu! je bande et je la fous'(40).

Comme le souligne P. Laroch, 'il s'agit bien ici de la 'conversion' d'un libertin mondain qui se proclame'libertin de qualité' en rejetant toutes les règles du libertinage, à commencer par la première: le caractère gratuit et inconséquent des aventures galantes [...].'17

Plus question de beaux discours et de belles phrases ('Vous pensez bien que nous ne parlons pas sentiment. Est-ce qu'elle s'embarrasse de ces foutaises-là?'(56)), c'est l'argent seul qui compte.

La linéarité du roman libertin traditionnel ${ }^{18}$ (succession d'aventures galantes) est conservée, mais le catalogue des conquêtes se transforme en liste des clientes; ${ }^{19}$ la structure narrative se maintient mais tout est perverti, converti: la chasse au plaisir et aux maîtresses devient synonyme de ruée vers l'or! C'est lui qui désormais fera courir l'aristocrate. La noblesse, qui méprise la bourgeoisie et toute forme de commerce, se prostitue ou achète un corps qui lui procurera du plaisir, Auri sacra fames, ${ }^{20}$ et se livre ainsi au plus vil des échanges.

$\mathrm{D}^{\prime}$ aucuns pourraient rétorquer, avec raison, que Mirabeau lui-même n'était pas, loin de là, un modèle de désintéressement. Ses dettes sont 
célèbres, ses fastueuses dépenses le sont tout autant. Il fut toute sa vie dépendant de l'argent, ${ }^{21}$ plus souvent pour vivre selon ses désirs que pour survivre.

Il ne s'agit là que d'une contradiction apparente. En effet, bien souvent, Mirabeau s'identifie à son personnage. ${ }^{22}$ Beaucoup des réflexions du libertin de qualité semblent sortir de la bouche de l'auteur. Nous avons dès le début de cette analyse parlé d'ironie, et donc de distance. Mirabeau fait preuve d'une admirable autodérision; il connaît ses points faibles et les raille. Et sa démonstration n'en est que plus brillante.

Nous en arrivons maintenant au deuxième volet de cette analyse: la peinture de moeurs et la satire sociale.

Le catalogue des clientes du libertin de qualité constitue une véritable galerie des femmes. 'Tu ne saurais croire combien ce cadre, qui ne semble rien, amène de portraits et de contrastes plaisants; toutes les sortes de femmes, tous les états y passent tour à tour: l'idée en est folle, mais les détails en sont charmants [...], ${ }^{23}$ explique-t-il à Sophie. 'C'est, sous une écorce très polissonne, une peinture vivante, et même assez morale, de nos moeurs et de celles de tous les états. Les femmes de cour, les religieuses et les moines y sont surtout traités à souhait. ${ }^{24}$

Mirabeau n'épargne personne, de la soubrette à la duchesse, de la putain de l'opéra à la dévote. Mais, la citation ci-dessus le laisse deviner, ce sont surtout les femmes de cour, les privilégiés et l'institution religieuse qu'il égratigne avec le plus de délectation et de virulence.

Mirabeau distille ses critiques par petites touches au fil des pages. Ainsi, on trouve en de nombreux endroits diverses allusions aux moeurs de la cour. Une duchesse prend un prince pour amant en titre, mais préfère batifoler avec ses laquais: 'c'est le pain quotidien d'une duchesse,' précise-t-il ${ }^{25}$ les grands tirent vanité de leurs titres et de leur naissance bien souvent sans raison:

[à propos d'un baron de province] Pour de l'esprit, son arbre généalogique l'a dispensé d'en avoir. Grand lecteur de gazettes, grand politiqueur, se faisant monseigneuriser par ses valets, par un curé qui, ainsi que lui, fait pour toute érudition marquer un cent de piquet; mangeant peu, dormant moins, et jaloux comme un tigre d'une jolie personne que trois mots de latin avaient baronisée (116).

Ou encore: 'Apprenez, monsieur le bougre, qu'il n'y a point de sot métier quand il nourrit son maître; et que de grands noms dans la France ne tirent leur illustration ou leur fortune que du cul d'une putain...' (126).

L'un des personnages se révèle un véritable archétype de la 'morale' de la haute aristocratie: la marquise de Vit-au-Conas. Avec elle tous les masques tombent, les illusions s'envolent. Tout comme Versac dévoilait 
à Meilcour les rouages de la vie de cour, Madame de Vit-au-Conas décape en profondeur le vernis de la bonne société pour laisser paraître les vraies valeurs qui la régissent.

Personnage des plus originaux (elle est pourvue des deux sexes et paie le narrateur pour qu'il accepte de se laisser sodomiser!), intrigante et dissimulatrice, elle a acquis à la cour 'le caractère de son état' (71); elle a compris que la perfidie y est la clef de toutes les réussites. Pour les femmes comme elle, le libertinage est une habitude que seule la diversité des amants rend agréable. A les fréquenter, on risque d'attraper 'ce qu'on [leur] a communiqué sans scrupules.' Elles rejettent en bloc ce qu'on nomme morale, mais en adoptent le fard.

Laissons-là s'exprimer elle-même sur ces matières:

Le sentiment est regardé parmi nous comme une chimère, nous en parlons avec emphase, avec esprit, raffinement même, précisément parce qu'il ne nous a jamais touché.[...] La pudeur est grimace, la décence hypocrisie, les qualités se dénaturent, les vertus sont chargées des couleurs du vice; mais la mode, les grâces embellissent tout, on ne prise l'esprit que par le jargon qui l'accompagne. En un mot, c'est de nous que dépend la fortune, et nous sommes aussi aveugles qu'elle [...] Prends donc un extérieur hardi, impertinent même dans le tête-à-tête; brusque tes aventures, tu ne serais téméraire que dans le cas de faiblesse, et le seul manque de respect que nous ne pardonnions pas, c'est une faute d'orthographe (73-74).

A la cour, tout n'est que tromperie et dissimulation, vice et corruption. Rien ne choque autant ces dames qu'une faute d'orthographe: la futilité et la vanité remplacent chez elles toute forme d'idéal.

On pouvait difficilement aller plus loin dans la satire. Il y parviendra pourtant, quand il attaquera les privilégiés et l'institution religieuse.

Comme c'est souvent le cas au XVIIIe siècle, on ne trouve chez Mirabeau aucune trace d'antireligiosité (l'épître à Satan est bien plus une bouffonnerie qu'une profession d'incroyance). Ce qu'il attaque, avec une férocité absolue, ce sont les voeux forcés, la vie 'dénaturée' des religieuses et des moines, et, surtout, le pouvoir de l'Eglise en tant qu'institution politique.

La critique des voeux forcés et de la vie monastique n'est pas un élément neuf dans le roman du XVIIIle siècle. Il s'agit au contraire d'un thème récurrent, traité avec plus ou moins de succès tant par des auteurs aussi célèbres que Diderot que par des écrivains aujourd'hui complètement oubliés. Mirabeau n'échappe pas à la règle. Défenseur de la Liberté et de la Nature, il ne pouvait s'abstenir de lancer une charge violente contre l'enfermement contre-nature des religieux, qui dérègle les sens et l'esprit. Fatigué de la vie tonitruante de la capitale, le libertin de qualité 
se rend dans la Marne où il fait un séjour à l'Abbaye de ${ }^{* * *}$. Il n'y observera pas, on s'en doute, le voeu de chasteté. Il commence par l'abbesse aux pâmoisons sensuelles, pour finir par coucher avec toute l'abbaye, et ... en tirer un profit considérable ('[...] je partis chargé d'or et de sucre'(112). En effet, rappelons-le, c'est le motif de l'argent qui sert d'articulation à tous les épisodes du roman.

Cette retraite dans la maison de Dieu est bien évidemment le prétexte à de nombreuses réflexions. Cet extrait fera juger de la force des expressions, de la concision des formules qui rendent le texte percutant sans emphase, impitoyable mise à nu de la vie claustrale et réécriture des saintes 'extases':

Vous ne connaissez pas, mon ami, les républiques femelles, dont l'abbesse est comme le doge. La plupart des filles qui les composent ont été enrôlées malgré elles dans la milice céleste; on les a faites épouses d'un être immatériel, et les charmes de la contemplation ne détruisent pas en elles la corporalité. Il en résulte dans la jeunesse une révolte des esprits charnels, un conflit de juridiction entre le sens et la raison, entre le créateur et la créature, où souvent la faiblesse humaine est obligée, comme Pilate, de s'en laver les mains. Tout cela ne fait que tromper les passions, irriter les désirs, les allumer davantage... De là les nerfs, les spasmes, etc. Dans la vieillesse, on est pie-grièche, colère, âpre, grondeuse. De là encore les inspirations, les apparitions et toutes les folies que les uns ont brûlées, les autres canonisées... [...] On ne peut pas toujours prier; il faut médire; prendre son prochain par les pieds et par la tête: le tout pour son bien, et la plus grande gloire de Dieu.[...] Que serait-ce si, te racontant mille scènes dignes de l'Arétin, je t'effrayais de la corruption que ces demoiselles vont puiser, jusqu'au moment où on les marie, dans ces lieux consacrés à la vertu et prostitués aux vices? Eh! que serait-ce encore si je te traçais les scènes du désespoir qui se passent dans le secret et le silence? Les brigues, les trahisons, les complots, tout ce qui doit nécessairement enfanter la crainte, la servitude et la barbarie? (110-111)

C'est l'homme des Lumières qui parle, bientôt rejoint par celui qui sera le tribun de la Révolution, criant haro sur l'inutilité des prêtres que $l^{\prime}$ Etat entretient ${ }^{26}$ ('Partisans de la population! Bande-à-l'aise économistes $!^{27}$ Est-ce un foutu calcul de morts ou de naissances qui donnera des enfants à l'Etat? Tous vos abbés, ennuyeux raisonneurs, et qui manquent de couilles, ont des pensions, tandis que j'use mon vit sans fruit et sans honneur.'(136)), et sur les privilèges qui font qu'un homme riche, ayant obtenu une charge ecclésiastique, devient immédiatement directeur des âmes et parangon de vertu: 'Mon instituteur était un Monseigneur à qui un bon évêché et deux abbayes, affermées cent mille francs, donnaient le privilège de prêcher la vertu chez les filles de la capitale, ou chez les titrées de la cour; ce qui revient au même'(82). 
Comme avec la Marquise de Vit-au-Conas, Mirabeau a choisi, avec un certain didactisme, de concentrer sur un même personnage tout son anticléricalisme: le Père Ambroise, symbole lumineux du pouvoir de l'Eglise en tant qu'institution et que puissance politiques, dont le discours permettra à l'auteur de renouer une fois encore avec l'esthétique du dévoilement.

L'espace narratif consacré au faux dialogue ${ }^{28}$ entre le Père Ambroise et le narrateur est significatif. Plus de douze pages couvrent cet épisode particulièrement important. Mirabeau y laisse libre cours à sa verve acérée contre le pouvoir de l'Eglise, qui aveugle les fidèles et les contient dans la peur. Le meilleur Voltaire n'atteint pas à plus de virulence.

Adoptant un style proche du pamphlet, il dénonce la lubricité des hommes d'Eglise ('Ils ne font rien d'autre que le narrateur: je dirige cons et consciences' (142)), leur hypocrisie ('L'hypocrisie nous est [...] salutaire et nécessaire,' (138)), et leur cupidité ('Mille fraudes, mille tours de passe-passe nous aident à leur escroquer de l'or qui, décorant les autels de la superstition, alimente les supports du vice; car foutre je suis de bon compte, à commencer par moi, nous ne valons rien' (141)).

Il attaque encore leurs abus d'autorité (142,143-144), puis passe à une longue dissertation dans laquelle le Père Ambroise explique à notre libertin, abasourdi, le fonctionnement de l'Eglise et dépeint les moeurs de ses serviteurs.

Une remarque s'impose. Nous avons vu que le narrateur faisait profession d'amoralité absolue, que l'amour de l'argent avait chassé en lui tout préjugé, et, cependant, il se montre profondément choqué, effrayé par les paroles du Tartuffe. A travers lui, Mirabeau exprime toute son angoisse devant le pouvoir d'une Eglise corrompue qui n'a plus rien à voir avec la spiritualité, pieuvre redoutable dont les tentacules étranglent le monde. C'est 'à la hache que Mirabeau dissocie l'Eglise de la Bible,' souligne avec justesse Charles Hirsch dans sa préface aux Oeuvres érotiques de Mirabeau.

De la soumission des peuples par la crainte aux armes de l'Eglise, de la puissance politique à la vénalité des Croisades, des guerres de religion aux complots et aux crimes... rien n'est épargné, tout est dévoilé dans une langue claire et puissante. Véritable morceau d'anthologie, cet extrait mériterait qu'on le cite en entier. Nous n'en donnerons ici qu'un bref aperçu:

Tu n'ignores pas qu'il fut un temps où la crasse ignorance enveloppa le monde; le fanatisme et la superstition régnèrent en adeptes sur ses siècles heureux... Age à jamais mémorable et fortuné où le froc commandait au diadème, où les Bernard, les François, les Dominique, [...] savaient émouvoir, exalter la bile de l'imbécile chrétienneté (sic)! Prophètes audacieux et menteurs, ils entassèrent des 
millions de croisés dans les sables de l'Egypte et de la Palestine; et l'Europe, à leur premier signe ébranlés contre l'Asie, courut y chercher de vastes tombeaux, tandis que ses crédules habitants devenus nos vassaux, laissaient dans nos mains assez de dépouilles pour élever la vraie Jérusalem;[...] Alors tout moine était saint; tout homme un peu éclairé au-dessus de son siècle excommunié.[..] Heureux temps! ils changèrent, hélas! ... La philosophie parut; non pas cette tracassière verbeuse qui se traîne encore en rampant dans la poussière de l'Ecole; mais cette lumière vive et fatale qui a dissipé les vapeurs du fanatisme, et brisé les hochets de la superstition; tels les oiseaux de nuit, nous fûmes blessés par l'éclat du jour.[...] Nous avions à nous venger; du fond de nos asiles, nous soufflâmes la discorde, nous fomentâmes ces guerres civiles qui ont inondé de sang l'Europe déchirée; nos libelles, nos serments séditieux, les séductions du confessionnal nous suffirent pour aiguiser les poignards; et grâce à nos efforts, il fut universellement reconnu qu'il est permis, qu'il est saint de tuer un hérétique; $c^{\prime}$ est-à-dire notre ennemi.[...] Ici, les fils de saint Dominique font périr le dernier des Valois: là, ceux d'Ignace immolent Henri, que des philosophes osent encore pleurer; les bûchers, le fer, les poisons nous servent tour à tour, les victimes s'amoncellent, les bourreaux et les assassins sont fatigués; les prisons regorgent d'innocents, et nous de sang, d'or et de volupté (147-149).

Ces phrases terribles résonnent encore de colère. Le grotesque fait place à la vérité nue; on ne peut, aujourd'hui encore, s'empêcher de trembler. Apologiste de l'ignorance qui a soumis les peuples, le Père Ambroise s'en prend enfin avec véhémence à la Philosophie qui a fragilisé l'emprise de l'Eglise sur les esprits:

Une révolution, éloignée peut-être, mais certaine, menace de nouveau le monde; nous foulerons aux pieds ces hommes superbes qui osent nous dédaigner; nous commanderons encore... Puissions-nous replonger les humains dans la barbarie, anéantir les sciences, arracher jusqu'au germe funeste de cette philosophie perfide qui nous abreuve d'humiliations; élever enfin sur tant de ruines le nouvel édifice de notre grandeur![...] Non, nous ne craignons rien de ce siècle vénal, nous payons des protecteurs qui deviendront nos esclaves, ils nous rendront au centuple ce qu'ils nous auront coûtés (150).

Nous en arrivons maintenant à la dernière partie de cet article, consacrée aux aspects purement linguistiques et littéraires qui soutiennent le récit.

La langue et le style se révèlent en effet deux éléments essentiels du roman. Mirabeau ne s'est pas contenté de critiquer la société de son temps entre deux scènes pornographiques. L'éloquence du tribun et l'art de l'écrivain se trouvent ici mis au service de la satire et du grotesque. 
La crudité des expressions, la gouaille qu'il manipule sans interruption d'un bout à l'autre du roman, associées à un style léger et alerte, à un rythme vif et gaillard, confèrent au roman tout son charme et participent de sa nouveauté. Malgré le vocabulaire graveleux des scènes 'érotiques' et des réflexions du narrateur, jamais on ne tombe dans la grossièreté et la platitude.

'Tout ce que je puis te dire, c'est que c'est une folie singulièrement neuve, et que je ne puis pas relire sans rire, ${ }^{29}$ pourrions-nous dire avec Mirabeau. Ma conversion est en effet un texte plein d'humour, renforcé par le baroque des descriptions. Tout y est outré, tout y est démesure et exagération. Ces deux traits stylistiques (humour et baroque) sont omniprésents et appuient le discours.

Véritable acrobate de la langue et homme instruit des littératures anciennes et modernes, Mirabeau fait rire. Il a l'art de la formule, ${ }^{30}$ n'hésite pas à recourir aux jeux de mots et phrases à double sens, ${ }^{31}$ et truffe son texte de citations littéraires détournées de leur signification originelle. ${ }^{32}$

D'une manière moins ponctuelle, les descriptions des clientes constituent de véritables tableaux baroques. Surcharges de détails, profusion de couleurs et d'attitudes, outrance dans la laideur, le tout assaisonné du ridicule de la situation: telles sont les caractéristiques de ces peintures sans pitié, qu'on ne peut lire sans sourire. Ainsi, par exemple, le portrait de Madame In Aeternum, dont le nom est déjà tout un programme:

Peignez-vous, mon ami, un vilain enfant de quelque soixante ans. Sa figure est un ovale renversé, une perruque, artistiquement mêlée avec un reste de cheveux reteints en noir, en ombrage la pointe, des yeux rouges et qui louchent pour se donner un regard en coulisse, une bouche énorme, mais que Bourdet a fort bien meublée, du blanc, du rouge, du vermillon, du bleu, du noir, arrangés avec un art, une symétrie que des yeux connaisseurs et un odorat exercés peuvent seuls découvrir (58).

et plus loin (62-63):

Ma déesse était en cornette... Sacredieu, qu'elle avait d'appas! Son lit à la turque, de damas jonquille, semblait assorti à son teint [...]. Un sourire qu'elle grimace me fait apercevoir qu'elle ne mord point. Enfin, je grimpe sur l'autel.[...] Je parcours des mains et des pieds les vieux charmes de ma Dulcinée... de la gorge... je lui en prêterais au besoin... Des bras longs et décharnés, des cuisses grêles et desséchées, une motte abattue, un con flétri et dont l'ambre, qui le parfume, à peine affaiblit l'odeur naturelle...Enfin, n'importe, je bande; je ferme les yeux; j'arpente ma haridelle, et j'enfourne. [...]Une bosse de grandeur hon- 
nête que je viens de découvrir me sert de point d'appui pour l'autre main. Son cou tendu $\mathrm{m}$ 'allonge un déplaisant visage qui, gueule béante, $\mathrm{m}^{\prime}$ offre une langue appesantie, que j'évite par une forte contraction de tous les muscles de ma tête. Enfin, je prends le galop... Ma vieille sue dans son harnois; sa charnière enrouillée s'électrise, et me rend presque coup sur coup; ses bras perdent de leur roideur, ses yeux se tournent; elle les ferme à demi, et réellement ils deviennent supportables... Sacredieu,j'enrage; cela ne vient pas; je la secoue...et tout à coup la bougresse m'échappe... Foutre, la fureur me prend, je m'échauffe; le talon tendu contre une colonne, je la presse, je l'enlève; la voilà qui marche...

Nous assistons à une réelle frénésie linguistique, caractéristique de l'écriture dans laquelle est composé le roman. Un peu plus loin, lorsqu'il introduira la discussion sur l'opéra, Mirabeau nous donne une véritable métaphore de ce style baroque, voire du roman lui-même:

Oui, il faut du chromatique, beaucoup de fausses quintes; on relève cela par l'accord parfait; déjà on passe dans le ton relatif par la tierce mineure: appuyezmoi une septième diminuée. Si le mode est mineur, grimpez au majeur; semezmoi des bémols; [...]ensuite un choeur en fugue à deux sujets bien sortants l'un de l'autre, parce que cela marque la dispute, le conflit de juridiction, surtout que cela crie comme le diable[...]; ensuite un grand silence; c'est imposant ça, hein? ...Un trois temps bien tendre pour faire le contraste (152-153).

'Faire le contraste,' voilà bien le mot. Ma conversion est une suite de contrastes: contrastes de tons et de propos, mais aussi de langues et de styles d'écritures. A côté de l'humour et du baroque, on trouvera le lyrisme préromantique. Certes il faudra le lire comme le reste, avec distance, mais il est bel et bien présent.

Après avoir perdu une forte somme au jeu, le libertin de qualité décide de quitter Paris. Il se perd dans une rêveries sur les beautés de la nature, abîmée par l'artifice (96-97). Le passage est étonnant: le narrateur se fait l'espace d'un instant héros romantique en quête de paysages sauvages, préservés de l'industrie des hommes:

La main de l'architecte a décoré ces salles tristement superbes; la règle impérieuse a tracé leurs contours; la serpe, la faux ont mutilé les dryades gémissantes pour arrondir ces colonnes ou former des amphithéâtres. J'entends le bruissement des eaux... Hélas! la naïade en pleurs n'y roule point ses flots argentés; mille canaux emprisonnent cette onde; des formes bizarres, des bouches d'airain l'élancent dans les airs; elle retombe brisée dans ces bassins où elle se perd sans pouvoir arroser le bocage qui la désire...O hommes! votre despotisme réduira donc tout à l'esclavage! 
Le paragraphe suivant semble être extrait de Rousseau ou même de Chateaubriand! Rêveur solitaire, perdu dans ses pensées, il devient la caricature du héros romantique:

Que je suis loin, grand Dieu! de cette douce mélancolie, où l'âme attendrie perd le sentiment douloureux de ses peines! où des larmes involontaires, mais précieuses, dégonflent le poitrine oppressée, et rafraîchissent la paupière!... Je suis sombre: mes pensées tumultueuses s'agitent, se choquent, se confondent; je reviens à pas lents, l'air rêveur, la tête penchée... [...] Je reviens à la ville. Toute la vitesse de mes chevaux ne me sert pas à mon gré: je suis à peine arrivé que je voudrais être ailleurs; je cherche avec ardeur des objets nouveaux...Ah! il n'en est point qui puissent guérir un coeur blasé sur tout. ${ }^{33}$

Ce roman malheureusement trop peu connu offre donc un intérêt incontestable. Mirabeau y excelle dans l'art du grotesque et de la fresque baroque. Il parvient à la fois à faire rire et réfléchir sur des sujets particulièrement graves.

Le texte n'a pas vieilli et est toujours accessible au lecteur du XXe siècle. Intemporalité des thèmes? Sans doute, mais aussi admirable talent de l'écrivain qui unit les qualités du style à la vigueur de la langue.

Ma conversion est une sorte de miroir déformant, qui gomme les apparences et fait apparaître sous des couleurs plaisantes les moeurs $\mathrm{d}^{\prime}$ une France en pleine mutation.

C'est un texte multiple qui a mis en évidence la complexité du personnage de Mirabeau. Homme des Lumières et artisan de la Révolution, il est le témoin d'une époque charnière, où se côtoient tragique et fantaisie, conservatisme et progrès.

Ses discours (son éloquence les personnalisa, même s'ils doivent beaucoup à d'autres) et ses traités ont vu s'exprimer le politique, $M a$ conversion a distrait le prisonnier de Vincennes, tout en lui permettant de dénoncer une société tout entière: celle de l'Ancien Régime finissant, mais aussi celle de l'argent que verra s'épanouir le XIXe siècle bourgeois.

\section{VALÉRIE VAN CRUGTEN-ANDRÉ}

Université libre de Bruxelles 


\section{Notes}

1 B. Didier, La littérature de la Révolution française, (Paris, P.U.F., 1988) 3.

2 On pense tout particulièrement aux travaux de Béatrice Didier, de Michel Delon ou de Robert Darnton.

3 C'est du moins l'opinion de la critique traditionnelle, qui définit le roman libertin comme un 'roman de la bonne compagnie', mettant en scène des personnages issus de l'aristocratie. Ainsi, pour L. Versini ( Laclos et la tradition (Paris:Klincksieck,1968) ou R. Abirached (art. libertin de l'Encyclopedia Universalis), pour ne citer qu'eux, le roman libertin naît avec les romans de Crébillon fils et disparaît avec le chef-d'oeuvre de Laclos. On trouvera une réflexion terminologique complète sur la notion de roman libertin dans ma thèse de doctorat, à paraître chez $\mathrm{H}$. Champion.

4 qu'il 'emprunta' largement à quelques-uns de ses amis!

5 On doit aux éditions Fayard l'édition complète des Oeuvres érotiques de Mirabeau, dans la collection 'L'Enfer de la Bibliothèque nationale' (Paris,1984).

6 Nous n'avons trouvé aucune trace de roman antérieur dont le narrateur, à l'image $\mathrm{du}$ libertin de qualité, clamerait, dès les premières lignes, son appartenance au monde de la prostitution et ferait au lecteur le récit de ses aventures dans le métier. Apollinaire, dans son 'Introduction, essai bibliographique, analyse et notes' de L'oeuvre du comte de Mirabeau (Paris:Bibliothèque des Curieux,1910) écrit dans ce sens: ' $C$ 'était la première fois sans doute que l'on faisait un personnage romanesque de l'homme qui vit au dépens des femmes.' En réalité, ce qui est neuf, ce n'est pas le fait que le personnage vive aux dépens des femmes (Cf. COULET (H.), 'Mirabeau romancier libertin,' in Mémoires de l'Académie des sciences, agriculture, arts et belles-lettres d'Aix (Le bicentenaire de la mort de Mirabeau à l'Académie d'Aix. 2 avril 1791-2 avril 1991) (Aix-en-Provence:Académie d'Aix Musée Arbaud, Nouvelle série - tome 4,1991, 37) mais bien qu'il s'adonne à la prostitution proprement dite.

7 Mirabeau avait déjà bien souvent tâté de la paille des cachots, grâce aux bons soins de son père. Cela lui inspira une importante réflexion sur l'arbitraire des lettres de cachets, Des lettres de cachet et des prisons d'état, qu'il composa dans cette même prison.

8 En réalité, il ne s'agissait pas d'un enlèvement mais bien d'une fuite à deux. Nous renvoyons, pour plus de détail à G. Chaussinand-Nogaret, Mirabeau (Paris:Seuil,1982).

9 Lettres à Sophie (Paris:Brissot-Thivars,1826) t.3:345.

10 Lettres 345.

11 Lettres 335.

12 Lettres 345.

13 Lettres 345.

14 Oeuvres érotiques de Mirabeau 173. A partir, de maintenant, les numéros de pages qui suivent les citations du roman renvoient à cette édition.

15 Cf. H. Coulet, 'Mirabeau romancier libertin,' op.cit. Il écrit aux pages 36-37:

'Celui-ci [le narrateur], encore une fois désespéré, veut entrer à la Trappe, en est détourné par des amis, décide de se 'convertir' (d'où le titre du roman) en se mariant $[\ldots]]^{\prime}$ 
16 Remarquons que la nouveauté vient aussi du fait qu'il s'agit d'un homme qui se prostitue et non d'une femme.

17 P. Laroch, Petits-maîtres et roués. Evolution de la notion de libertinage dans le roman français du XVIIIe siècle, (Québec: Presses de l'Université Laval, 1979) 152.

18 Ce que J.P. Dubost appelle 'parataxe libertine' dans son Eros und Vernunft. Literatur und Libertinage (Frankfurt: Athenäum,1988).

1944 sqq., on assiste à la séduction d'une dévote. La scène rappelle, en ton majeur, les machinations de Valmont pour séduire la présidente de Tourvel. Cependant, ici, la jouissance suprême consiste bien plus à se faire payer par la dévote qu'à vaincre sa pudeur.

20 Exergue présent sur la page de titre de l'édition de 1784.

21 Il espérait d'ailleurs gagner de l'argent grâce à ce roman, cf. Lettres à Sophie 3:345.

22 Cf. Lettres à Sophie 3:334-335: 'Le premier alinéa te donnera une idée du sujet, et t'apprendra en même temps quelle fidélité je te prépare.[...]' et 345:'Depuis les femmes de cour, qui y sont cavées à fond, j'ai fini les religieuses et les filles d'opéra; j'en suis par occasion aux moines; de là je me marierai, puis je ferai peut-être un petit tour aux enfers (où je coucherai avec Proserpine), pour y entendre de drôles de confessions...'

23 Lettres à Sophie 3:344-345.

24 Lettres 352.

25 Oeuvres érotiques de Mirabeau 81. On retrouve là une idée déjà présente dans Margot la Ravaudeuse de Fougeret de Monbron. Margot, devenue grande dame, prend son plaisir avec ses laquais.

26 On ne peut s'empêcher de lire ici une allusion à l'obsession populiste du XVIIIe siècle. On craignait en effet de voir s'accroître la dépopulation. Cf. J.-P. Aron et R. Kempf, La Bourgeoisie, le sexe et l'honneur (Bruxelles:Complexe,1984).

27 Dans cette tirade, c'est son père, l'Ami des hommes, que Mirabeau attaque en réalité.

28 Les interventions du libertin de qualité servent en réalité de charnières aux différents arguments énoncés par le Père.

29 Lettres à Sophie 345.

$30 \mathrm{Cf}$. à propos d'un cocu:'La lecture en apprit au mari plus qu'il n'aurait voulu, et il se reconnut de la manière la plus claire Chevalier du Croissant'(114)

31 Cf. à propos de la Marquise de Vit-au-Conas: 'Le mari de la marquise était à son régiment; il lui laissait du vide. Je m'offris à le remplir'(68), et 'Je me rendis chez la Vit-au-Conas: elle était en possession de mes jours de congé; d'ailleurs nous avions contracté une amitié commode. Ô que cette femme-là gagne à être approfondie!(81)

32 Il découvre le 'braquemart de huit pouces'(70) de la marquise: 'En deux tours de mains j'arrange la marquise; je me précipite...Dieux!...le flot qui m'apporta reculé épouvanté' (70) et, après que la marquise l'a sodomisé: 'A près ce bel exploit, la marquise m'apostrophe: - Rodrigue, qui l'eût cru! Et moi, en portant la main au pauvre blessé, et faisant piteuse grimace:-Chimène, qui l'eût dit?'(72). Ou encore, lorsqu'il découvre que sa femme n'est plus vierge: 'O rage! ô désespoir! moi la terreur des maris!'(171)

33 On verra encore, dans le même esprit, l'attrait des cimetières et l'idée de la mort. 